\title{
Parental smoking and lower respiratory illness in the first three years of life
}

\author{
D. M. FERGUSSON, L. J. HORWOOD, F. T. SHANNON, AND BRENT TAYLOR \\ From the Department of Paediatrics, Christchurch Clinical School of Medicine, Christchurch, New Zealand
}

SUMMARY The relationships between parental smoking and the rates of lower respiratory illness during the first three years of life were examined for a birth cohort of 1265 New Zealand children. Lower respiratory illness varied significantly with maternal smoking for the first year; there was equivocal evidence of a relationship between maternal smoking and lower respiratory illness in the second year; and by the third year the relationship had clearly disappeared. Paternal smoking had no significant effect on rates of lower respiratory illness at any time.

Application of logistic regression showed that for the first year rates of lower respiratory illness were approximately linearly related to maternal smoking: increases of five cigarettes a day resulted in an increase of 2.5 to 3.5 incidents of lower respiratory illness per 100 children at risk. Statistical control for maternal age, education, family size, and family living standards showed that the relationship between maternal smoking and rates of lower respiratory illness was not significantly influenced by these factors.

A number of studies have reported associations between parental smoking and lower respiratory illness in children. These reports have ranged from findings of increased rates of bronchitis and pneumonia in infants ${ }^{1-4}$ to increased morning cough and breathlessness in children of school age..$^{5-8}$ Most authors have been cautious about imputing a causal relationship on the basis of the correlational evidence, but at least three sets of findings suggest that the relationships are causal rather than coincidental.

Firstly, the association between parental smoking and childhood respiratory illness has been remarkably resilient to attempts at statistical control. Various studies have shown that the correlation persists when such factors as family social conditions ${ }^{124}$; family composition ${ }^{34}$; perinatal history ${ }^{2}$; breast-feeding ${ }^{4}$; lower respiratory illness in the child's family ${ }^{13}$; and the child's smoking habits ${ }^{8}$ have been taken into account.

Secondly, the correlation appears to be specific to lower respiratory illness and at least two studies ${ }^{3}$ have reported that a similar association does not exist for upper respiratory illness.

Finally, a study by Fergusson, Horwood, and Shannon ${ }^{4}$ indicated that the effects of smoking varied with the degree of contact between the smoking adult and the child: maternal smoking contributed significantly to lower respiratory illness in infants, but paternal smoking did not.
Despite the convergence of evidence suggesting a causal link between parental smoking and childhood lower respiratory illness, relatively little is known about the details of this association. In particular, information is absent or limited on the dose/response relationships which exist between parental smoking levels and rates of lower respiratory illness and the way in which the effects of parental smoking vary with the child's age.

In this paper we report on the results of a three-year prospective study of the effects of parental smoking on lower respiratory illness in children. The aims of the study were two-fold: to describe the way in which the effects of parental smoking on lower respiratory illness varied with the age of the child, and to provide estimates of the dose/response relationships between parental smoking levels and rates of lower respiratory illness.

\section{Method}

The data were collected during the first five stages of the Christchurch Child Development Study. ${ }^{9}$ In this study a birth cohort of infants born live in the Christchurch (New Zealand) urban region during the period 15 April 1977 to 5 August 1977 has been studied at birth, four months, one, two, and three years. At each stage information was collected by structured interviews with the child's mother, supplemented by information from hospital records 
and other sources. The topics examined by the interviews included the child's health, development, and family social and economic background.

Information on the medical history, including respiratory illness, of each child was collected in the following ways:

(1) During the periods birth to four months, one to two years, and two to three years each mother was provided with a diary in which to record her child's history of medical attendance. Compliance was good; $85 \%$ of mothers returned a diary record for the period from birth to four months and $60-65 \%$ for the periods between one and three years.

(2) At each interview the mother was asked to give details of her child's history of medical attendance. This information was obtained from the diary record where available or from maternal recall supplemented by telephone calls to the family doctor.

(3) The history of the child's hospital attendance was cross-checked against all available hospital records in Christchurch and by contacting hospitals outside the Canterbury region for relevant case information in other cases, subject to the signed consent of the mother. Comparison between maternal reports of hospital admission and hospital records revealed very good agreement between the two: only two mothers failed to report an admission for their child during the three-year period.

(4) Mothers were asked to provide data on the history of medical consultation, and were also questioned about the child's history of symptoms and illness which had not received medical treatment.

Using this information, it was possible to reconstruct the child's history of illness from birth to three years.

The following measures were used in the analysis:

(1) Lower respiratory illness. Two measures were constructed:

(a) Bronchitis/pneumonia-whether the child had attended a doctor or hospital for bronchitis, bronchiolitis or pneumonia during any of the periods birth to one year, one year to two years, and two to three years.

(b) Symptoms of lower respiratory illness-because measures of medically treated lower respiratory illness could have provided a biased estimate of morbidity, a second more general index was constructed. This was based on reports of medically treated bronchitis/pneumonia together with maternal reports of 'chesty cold' or 'wheezy chest' irrespective of whether medical treatment was sought. A child was defined as displaying symptoms of lower respiratory illness if he had received medical attention for bronchitis/pneumonia or if his mother reported 'chesty colds' or 'wheeze'. (Incidents in which chest wheeze was ascribed to asthma were not included in the definition).

(2) Parental smoking. At one year, two years and three years information was obtained on both maternal and paternal daily cigarette consumption.

(3) Control variables. The following measures were used in the analysis for the purposes of statistical control: maternal age, education, family size, and an interviewer rating of family living standards. (These variables were selected on the basis of preliminary tabular and multiple regression analyses of a larger set of possible control factors including birthweight, gestational age, sex, maternal ethnic status, family composition, family income, and early infant diet. This analysis showed that only maternal age, education, family size, and living standards were related to both parental smoking habits and lower respiratory illness in childhood).

RESPONSE RATES AND MISSING DATA

Table 1 shows the response rates for each year of data collection. Response rates remained uniformly high over the three-year period. Of the initial cohort of 1265 infants, 1143 remained in the study at three years. This represented $90 \%$ of the initial cohort and $96 \%$ of the cohort who were still alive and resident in New Zealand. In a few cases (10 to 15 a year) data were missing on parental smoking or history of lower respiratory illness. All analyses are based on the sample available in each time-period who had complete data on all variables in the analysis. The sample sizes involved are given by the N's shown in Table 2. Comparisons of the social and demographic characteristics of the sample (that is, maternal age, education, ethnic status, family income, socioeconomic status) between interviews showed no significant differences in sample composition arising from sample losses.

\section{Results}

Table 2 shows the annual rates per 100 children of bronchitis/pneumonia and total lower respiratory

Table 1 Response rates during the three-year study period

\begin{tabular}{llll}
\hline Time & $\begin{array}{l}\text { Number } \\
\text { interviewed }\end{array}$ & $\begin{array}{l}\text { \% original } \\
\text { sample (1265) }\end{array}$ & $\begin{array}{l}\text { \% of those alive } \\
\text { and resident in } \\
\text { New Zealand }\end{array}$ \\
\hline One year & 1180 & 93.3 & 96.8 \\
Two years & 1156 & 91.4 & 96.6 \\
Three years & 1143 & 90.4 & 96.4 \\
\hline
\end{tabular}


Table 2 Rates per 100 children of bronchitis/pneumonia and lower respiratory symptoms in the first three years of life by maternal and paternal smoking (number of children in brackets)

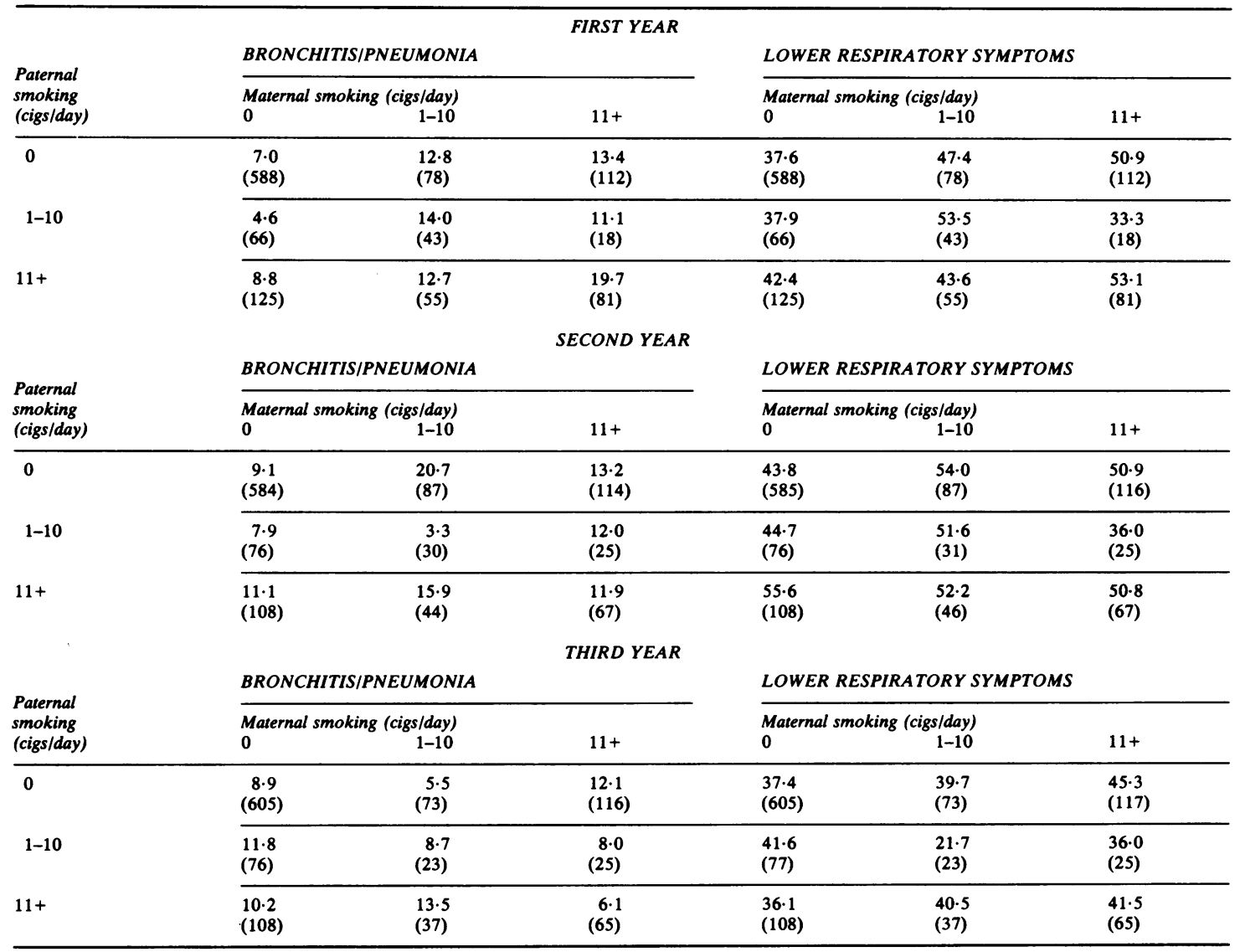

symptoms during the three-year study period. These rates are subdivided by maternal and paternal daily cigarette intake. A series of five log-linear models was fitted to the annual rate data to test for the presence of significant relationships between parental smoking behaviour and rates of lower respiratory illness. The method involved fitting a series of hierarchical models which differed from one another by one effect. From these models, tests of the contributions of maternal and paternal smoking behaviour to the rates of lower respiratory illness were derived from the differences between the values of the $\log$-likelihood $\chi^{2}$ statistic associated with each model. This analysis showed:

(1) For the first year, a model which assumed that the rates of childhood lower respiratory illness were independent of parental smoking did not fit the data. Further analysis showed that there was a significant tendency for rates of bronchitis/pneumonia $\left(\log \chi^{2}=17 \cdot 4 ; \quad\right.$ df $=2$; $\mathrm{p}<0.001)$ and of lower respiratory symptoms (log $\left.\chi^{2}=12.4 ; \mathrm{df}=2 ; \mathrm{p}<0.01\right)$ to vary with maternal smoking behaviour. Paternal smoking did not significantly contribute to the rates of lower respiratory illness either when considered alone or when maternal smoking behaviour was taken into account.

(2) For the second year, a model which assumed that the rates of lower respiratory illness were independent of maternal and paternal smoking behaviour was sufficient to fit the data for both bronchitis/pneumonia and total lower respiratory symptoms. However, for the bronchitis/pneumonia data it was found that a model which included maternal smoking as a factor related to lower respiratory illness resulted in a statistically significant improvement in the fit of the data over the model of the independence between the factors $\left(\log \chi^{2}=8 \cdot 3\right.$; df $=2 ; p<0.02)$. The results thus suggested an equivocal relationship between maternal smoking 
Table 3 Rates and differences in the rates per 100 children of bronchitis/pneumonia and total lower respiratory symptoms between children of non-smokers and smokers

\begin{tabular}{|c|c|c|c|c|c|c|}
\hline \multirow[b]{2}{*}{ Year } & \multicolumn{3}{|c|}{ BRONCHITIS/PNEUMONIA } & \multicolumn{3}{|c|}{ LOWER RESPIRATORY SYMPTOMS } \\
\hline & $\begin{array}{l}\text { Mother } \\
\text { Non-smoker }\end{array}$ & $\begin{array}{l}\text { Mother } \\
\text { Smoker }\end{array}$ & Difference & $\begin{array}{l}\text { Mother } \\
\text { Non-smoker }\end{array}$ & $\begin{array}{l}\text { Mother } \\
\text { Smoker }\end{array}$ & Difference \\
\hline $\begin{array}{l}\text { First } \\
\text { Second } \\
\text { Third }\end{array}$ & $\begin{array}{l}7 \cdot 1 \\
9 \cdot 1 \\
9 \cdot 3\end{array}$ & $\begin{array}{r}14.5 \\
14.2 \\
9.1\end{array}$ & $\begin{array}{r}7.4 \\
5.1 \\
-0.2\end{array}$ & $\begin{array}{l}38.4 \\
44.9 \\
37.6\end{array}$ & $\begin{array}{l}49 \cdot 1 \\
50 \cdot 8 \\
40 \cdot 6\end{array}$ & $\begin{array}{r}10.7 \\
5.9 \\
3.0\end{array}$ \\
\hline
\end{tabular}

and lower respiratory illness in the second year of life.

(3) For the third year, a model in which the rates of lower respiratory illness were assumed to be independent of maternal and paternal smoking was sufficient to fit the data for both measures. No significant improvement in the fit of the model was obtained by introducing assumptions about the dependence of lower respiratory illness on maternal or paternal smoking.

The major results of the log-linear analyses can be seen more clearly from Table 3 , which shows the differences in the rates of lower respiratory illness between children of smoking mothers and children of non-smoking mothers over the three-year period. During the first year, children of smokers had rates of lower respiratory illness which were seven to $11 \mathrm{per}$ 100 higher than for children of non-smokers; for the second year, this difference declined to five to six per 100 ; and by the third year the differences were clearly negligible ( 0 to 3 per 100$)$.

The results for the first year of life show that there is a general and significant tendency for rates of lower respiratory illness to rise with maternal daily

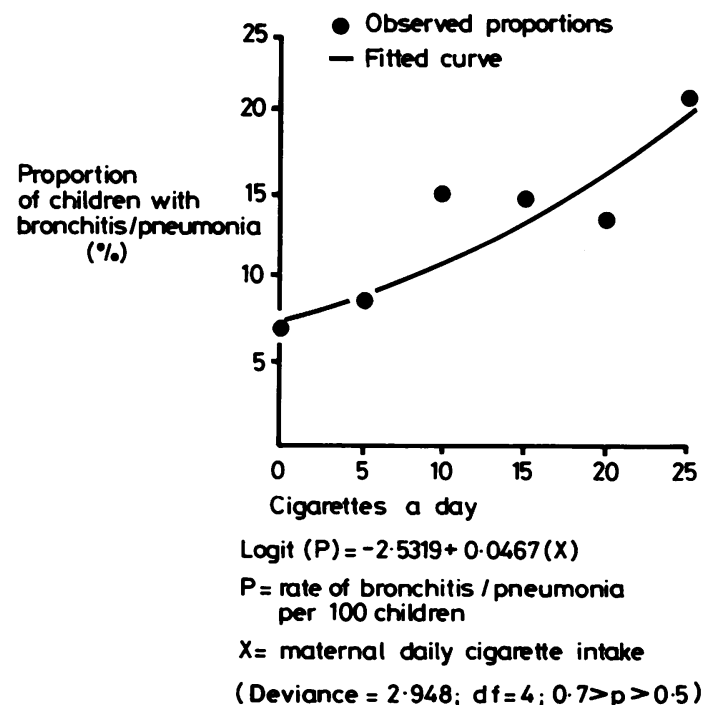

Fig. 1 Dose/response curve between maternal daily cigarette intake and rates of bronchitis/pneumonia in the first year of life. cigarette intake. However, because of the coarse grouping method used in Table 2, the nature of the relationship between cigarette intake and rates of illness is not clear. To provide a more sensitive analysis, the smoking behaviour of mothers was classified into six groups: non-smoker; one to five cigarettes a day; six to 10 cigarettes a day; 11 to 15 cigarettes a day; 16 to 20 cigarettes a day; and $21+$ cigarettes a day. Using the median intake values for these groupings, logistic models were fitted to the rates of bronchitis/pneumonia and total lower respiratory symptoms. The results of this analysis are summarised in Figs. 1 and 2, which show the fitted dose/response curves and the observed data. While both models showed a satisfactory fit $(0.7>p>0.3)$ to the data as measured by the deviance statistic, it is clear that there is some discrepancy between the fitted values and the observed data points. In particular, both analyses show a general tendency for the observed results to be higher than the predicted results for the group of subjects reporting an intake in the range of six to 10 cigarettes a day. Disregarding this discrepancy, the results suggest that increases of

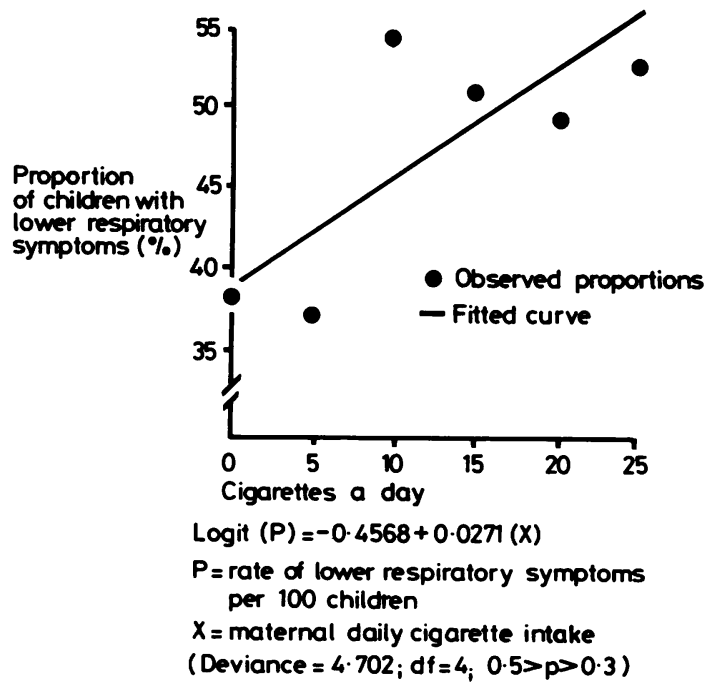

Fig. 2 Dose/response curve between maternal daily cigarette intake and rates of lower respiratory symptoms in the first year of life. 
Table 4 Comparison of slope coefficients of the unadjusted and adjusted fitted logistic curves

\begin{tabular}{lllll}
\hline & & & \multicolumn{2}{l}{ LOWER RESPIRATORY } \\
& \multicolumn{1}{c}{ BRONCHITISIPNEUMONIA } & \multicolumn{2}{l}{ SYMPTOMS } \\
& $B$ & SE & $B$ & $S E$ \\
\hline $\begin{array}{l}\text { Unadjusted } \\
\text { Adjusted }\end{array}$ & 0.0467 & 0.0112 & 0.0271 & 0.0076 \\
\hline & 0.0394 & 0.0118 & 0.0237 & 0.0081 \\
\hline & $\mathrm{t}=0.45$ & $\mathrm{p}>0.05$ & $\mathrm{t}=0.31$ & $\mathrm{p}>0.05$
\end{tabular}

five cigarettes a day result in an approximate increase of $2 \cdot 5-3 \cdot 5$ per 100 in the rates of lower respiratory illness.

However, the dose/response curves shown in Figs. 1 and 2 do not take into account the effect of other factors which may be correlated with both smoking and lower respiratory illness. To adjust the results for the possible effects of these factors, the data were re-analysed using logistic models in which maternal age, education, family size, and family living standards were introduced as additional factors. Table 4 gives a comparison of the $B$ (slope) coefficients for the unadjusted and adjusted models. The results show that the effect of introducing the control factors is to reduce slightly the slope of the regressions. However, the differences between the adjusted and unadjusted slopes are not significant, indicating that the dose/response relationships depicted in Fig. 1 provide a good approximation to the relationships after the data have been adjusted for confounding factors.

\section{Discussion}

In confirmation of the findings of Colley et $a^{1}$ the correlation between parental smoking and childhood lower respiratory illness appeared to be most marked for the first year of life and showed a steady decline with increasing age. At one year, clear differences between the offspring of smokers and non-smokers were evident; at two years the evidence was equivocal; and by three years an association between maternal smoking and infant lower respiratory illness was clearly absent. However, these findings are not entirely consistent with reports ${ }^{5-8}$ which have shown greater rates of respiratory problems (morning cough and breathlessness) among children of school age with smoking parents. One hypothesis which satisfies the available data is that the effect of parental smoking on childhood lower respiratory illness and symptoms is two-fold. During early life, and particularly the first year, prolonged contact with cigarette smoke would appear to precipitate or exacerbate lower respiratory illness in children; this effect is relatively short-lived and disappears at the age of about 2. However, prolonged exposure to cigarette smoke over a period of years may have a compound interest effect in compromising the respiratory function of children, with the result that by the time middle childhood is reached, children of smokers have a greater rate of lower respiratory illness or symptoms.

For the first year of life, the fitted dose/response curves showed an almost linear tendency for rates of illness to increase with maternal smoking: increases of five cigarettes a day resulted in increases in rates of lower respiratory illness in the region of 2.5 to 3.5 incidents per 100 children at risk. However, the observed data showed a number of deviations from the smoothed dose/response function. For both measures of lower respiratory illness the observed rates of lower respiratory illness for women reporting an intake of six to 10 cigarettes a day were markedly higher than the rate estimated from the curve. This aberration in the results almost certainly reflects the effects of a reporting bias in the daily cigarette intake estimates, with women having a tendency to round their estimates down to the convenient and perhaps socially acceptable ' 10 a day'. Because of the possible biasses in maternal reports, the dose/response curves should be treated as providing only an approximate measure of the sensitivity of rates of infant lower respiratory illness to maternal smoking habits.

This research was funded by grants from the Medical Research Council of New Zealand and the National Children's Health Research Foundation.

Reprints from Mr. D. M. Fergusson, the Christchurch Child Development Study, Department of Paediatrics, Christchurch Clinical School of Medicine, Christchurch Hospital, Christchurch, New Zealand.

\section{References}

${ }^{1}$ Colley JRT, Holland WW, Corkhill RT. Influence of passive smoking and parental phlegm on pneumonia and bronchitis in early childhood. Lancet 1974; ii: 1031-4.

${ }^{2}$ Harlap S, Davies AM. Infant admissions to hospital and maternal smoking. Lancet 1974; i: 529-32.

${ }^{3}$ Leeder SR, Corkhill R, Irwig LM, Holland WW, Colley JRT. Influence of family factors on the incidence of lower respiratory illness during the first year of life. $\mathrm{Br} J$ Prev Soc Med 1976; 30: 203-12.

${ }^{4}$ Fergusson DM, Horwood LJ, Shannon FT. Parental smoking and respiratory illness in infancy. Arch Dis Child 1980; 55: 358-61.

${ }^{5}$ Cameron R, Kostin JS, Zaks JM et al. The health of smokers' and non-smokers' children.J Allergy 1969; 43: 336-41.

${ }^{6}$ Colley JRT. Respiratory symptoms in children and parental smoking and phlegm production. $\mathrm{Br}$ Med $\mathrm{J}$ 1974; ii: 201-4.

${ }^{7}$ Norman-Taylor W, Dickinson VA. Dangers for children in smoking families. Community Med 1972; 128: 32-3.

${ }^{8}$ Bland M, Bewley BR, Pollard V, Banks MH. Effect of children's and parents' smoking on respiratory symptoms. Arch Dis Child 1978; 53: 100-5.

${ }^{9}$ Fergusson DM, Horwood LJ, Wright R, Stewart CR. Factors associated with planned and unplanned nuptial births. $N$ Z Med J 1978; 88: 89-92. 